rev.relac.int.estrateg.segur.6(1):113-125,2011

\title{
LA POLÍTICA DE SEGURIDAD EN COLOMBIA: ¿RUPTURA, O UNA CONTINUIDAD CREATIVA?
}

Vicente Torrijos Rivera*

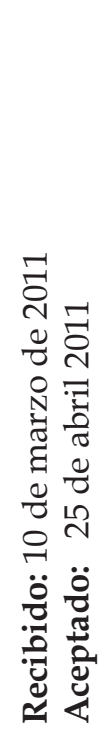

\section{RESUMEN}

La política de defensa y seguridad democrática (Psd) implementada por el presidente Álvaro Uribe Vélez ha sido objeto de diversas críticas y, al mismo tiempo, elogios. Como respuesta a los nuevos retos que debe asumir el presente gobierno de Juan Manuel Santos se escribe este artículo, el cual analiza dicha política -tanto fortalezas como debilidades- para así lograr establecer una serie de conclusiones respecto a su continuidad y los cambios necesarios que debe enfrentar para lograr adaptarse a las nuevas dinámicas del mundo y las transformaciones que se han generado al interior de las organizaciones guerrilleras como lo son las FARC y el ELN.

Palabras Clave: Política de Defensa y Seguridad Democrática (PSD) - Socialismo del siglo XXI - Diplomacia paralela Colombia - Estrategia - Seguridad.

* Profesor Titular de Ciencia Política y Relaciones Internacionales en la Universidad del Rosario, Bogotá, Colombia. tutoriascontorrijos@yahoo.com 


\begin{abstract}
The Democratic Defense and Security Policy (DDS) that was implemented by president Álvaro Uribe Vélez has been object to several critics, but at the same time, appraisement. This article was written as a response to the new challenges that the present government of Juan Manuel Santos has to face, it analyzes the mentioned policy - both its strengths as well as its weaknessesso that it may achieve a serious of conclusions about the continuity an the necessary changes that must be faced in order to adapt to the new dynamics of the world, and the transformations that have taken place inside the guerrilla groups such as the FARC and ELN.
\end{abstract}

Key Words: Democratic defense and security policy (Dds) Socialism of the twenty first centuryParallel diplomacy- Colombia- Strategy- Security.

\title{
RESUMO
}

A política de defesa e segurança democrática (Psd), implantada pelo presidente Álvaro Uribe Velez, tem sido objeto de diversas críticas e, ao mesmo tempo, elogios. Como resposta aos novos desafios que deve assumir o atual governo de Juan Manuel Santos, escreve-se esse artigo, que analisa tal política - tanto fortalezas como debilidades - para assim estabelecer uma série de conclusões no que diz respeito à sua continuidade e às mudanças necessárias que deve enfrentar para poderse adaptar às novas dinâmicas do mundo e às transformações, que têm sido geradas no interior das organizações guerrilheiras como é o caso das FARC e do ELN.

Palavras Chave: Política de Defesa e Segurança Democrática (PSD) - Socialismo do século XXI - Diplomacia Paralela- Colômbia - Estratégia - Segurança.

\section{PRIMERA PARTE: LA DIMENSIÓN INTERNA DE UNA POLÍTICA EN PERMANENTE CONTRASTE}

Uno de los defectos reiterados de la respuesta del Estado colombiano al desafío insurgente, encarnado por las organizaciones guerrilleras de las Fuerzas Armadas Revolucionarias de Colombia (FARC) y del Ejército de Liberación Nacional (ELN), ha sido la falta de continuidad y la improvisación de la estrategia contrainsurgente aplicada a efectos de su neutralización y contención.

Estos dos elementos han tenido un impacto muy importante y pueden considerarse variables independientes del fenómeno de la perpetuación y la longevidad de estos grupos armados ilegales. En efecto, a lo largo del tiempo, la respuesta estatal ha sido errática, oscilante, cortoplacista y, además, sujeta al vaivén de la coyuntura política y, con frecuencia, a las prioridades e intereses del gobierno de turno (García, 1992). 
En la actualidad, sin embargo, puede afirmarse que esas limitaciones endémicas están prácticamente superadas.

El diseño e implementación de la Política de Defensa y Seguridad Democrática -PSD- ("Política de Defensa y Seguridad Democrática," 2003) y la puesta en marcha de su estrategia de consolidación, por un lado, y la reelección del presidente Álvaro Uribe Vélez para un segundo periodo (2006-2010) y su sucesión en la persona del ex ministro de Defensa, Juan Manuel Santos, para el periodo 2010-2014, le han permitido al Estado colombiano construir y adoptar un marco estratégico para enfrentar la amenaza con un grado de sostenibilidad que explica en buena medida los logros obtenidos hasta ahora.

En ese sentido, se explica claramente la importancia que en el diseño e implementación de la PSD tuvo la existencia de un amplio consenso político y social a escala nacional en relación con:

(1) El agotamiento de la alternativa de la negociación, ante la contumacia de las FARC en el experimento de San Vicente del Caguán (Kline, 2007).

(2) La priorización de la lucha contra los grupos armados ilegales como condición indispensable para la supervivencia del sistema político (Rojas, 2009).

Ese consenso generalizado obedeció tanto a la expansión de la amenaza como a la sustancial deslegitimación que sufrieron las organizaciones armadas ilegales como consecuencia de la asfixia a la que condujeron los intentos de negociación en que llegaron a involucrar el gobierno (Pärssinen y Lammila, 2006).

A su vez, resulta innegable que en la elaboración de la estrategia de la PSD, el empoderamiento de las capacidades de la Fuerza Pública, la inteligencia recaudada en el Caguán y los recursos derivados del Plan Colombia constituyeron piezas fundamentales que, habiendo empezado a articularse bajo la administración Pastrana, potenciaron los esfuerzos del presidente Uribe desde el momento mismo de su posesión (Fundación Seguridad y Democracia, 2007).

Por otra parte, puede afirmarse que la PSD ha sido exitosa, reflejando en ese éxito una claridad estratégica que se deriva tanto de un profundo conocimiento del adversario (adquirido por vía de inteligencia), como de un amplio margen de acción (que le permitió al Gobierno Uribe sortear, con éxito, el chantaje humanitario de las FARC), a los que cabe añadir la innovación y la adaptación del dispositivo de seguridad y defensa del Estado (a través de la incorporación de nueva doctrina, como en materia de operaciones conjuntas, o de la acción integral basada en una mayor coordinación interagencial) (Rangel, 2007).

En ese sentido, el éxito de la PSD tiene que ver con la recuperación de la capacidad de respuesta del Estado, pero también con la capacidad para adaptarse a la dinámica de la confrontación, 
anticiparse al adversario creativamente y aprovechar las estructuras de oportunidad (en el plano político interno, en el plano comunicacional, en la escena y la coyuntura internacional) para maximizar su margen de autonomía y ensanchar su capacidad de desempeño.

De ahí que la sostenibilidad y la continuidad de la PSD no pueda medirse en términos de "inalterabilidad" sino, precisamente, en términos de su "flexibilidad adaptativa", la cual, en todo caso, presupone el mantenimiento del consenso generalizado en torno a la necesidad de bloquear militarmente la amenaza, condicionar cualquier tentativa de negociación a reglas precisas y no desacelerar el esfuerzo realizado.

Ello es así por cuanto aunque las organizaciones armadas ilegales están significativamente debilitadas, no por ello puede decirse que estén derrotadas, y los logros obtenidos hasta ahora no han alcanzado un punto definitivo de irreversibilidad

(Van Engeland y Rudolph, 2008).

Sin embargo, en tanto que las condiciones de seguridad han cambiado sustancialmente (y la amenaza se ha adaptado para sobrevivir, o se ha transformado y descompuesto como consecuencia de la creciente presión del Estado), es apenas lógico que cambie también, a fin de mantener su eficacia, la política de seguridad del Estado.

\section{Primera conclusión}

Por todas estas razones parece coherente que el Gobierno colombiano se muestre decidido a asegurarse el consenso político y social en materia de seguridad. Para ello dispone de enormes recursos de gobernabilidad en el marco de la coalición de "unión nacional" que rodea en el Congreso al presidente Santos, y cuya cohesión debe mantenerse y alimentarse en todos los asuntos relativos a la compleja problemática de seguridad. Y, sin duda, ese consenso implica la sincronía y concordancia de las élites y el reconocimiento del liderazgo presidencial para orientar el esfuerzo del Estado.

Puesto a rodar, al menos formalmente, ese consenso, el Gobierno exhibe ya la tendencia a capitalizarlo para validar y legitimar las adaptaciones que la estrategia de seguridad requiere en función de la transformación del clima de seguridad y de la capacidad de acción de la amenaza, significativamente baldada y, sin embargo, todavía perturbadora.

Esas adaptaciones deben suponer el ajuste del dispositivo contrainsurgente existente para enfrentar fundamentalmente dos escenarios: el derive terrorista de los grupos armados ilegales y/o su paulatina bandolerización como consecuencia de la erosión de su aparato organizacional, la "traquetización" de los cuadros medios, y el anquilosamiento que resulta del repliegue y a la hibernación forzosa a la que los obliga la necesidad de acumular fuerzas frente al envite del Estado. 
En consecuencia, y para asegurar la necesaria "flexibilidad adaptativa", el Gobierno parece entender la necesidad de redefinir metas y objetivos, factores de riesgo y de oportunidad, áreas de intervención prioritaria y roles y responsabilidades de las agencias involucradas.

En otras palabras, la continuidad de la PSD parece exigir, en el momento actual, su profunda reformulación: pero una reformulación no basada en expectativas triunfalistas, sino en el reconocimiento de que el impacto de lo logrado hasta ahora hace emerger un escenario de desafíos muy complejo, por un lado, y por el otro, que la desarticulación o eliminación de las organizaciones armadas ilegales no es sinónimo de superación de la violencia ni mucho menos del establecimiento definitivo e irreversible de un clima de paz absolutamente garantizado.

\section{SEGUNDA PARTE: LA DIMENSIÓN EXTERNA. EL VECINDARIO CRÍTICO EN PERSPECTIVA}

Durante los últimos años el conflicto interno ha sufrido importantes transformaciones que han alterado sustancialmente la dinámica de la problemática de la seguridad en Colombia. Una de esas transformaciones es, sin duda, su creciente y continua internacionalización, es decir, su mayor exposición e incorporación a la agenda de la política internacional así como su concurrente proyección más allá de las fronteras nacionales o transnacionalización (Fukumi, 2008).

Este proceso es resultado tanto de algunos fenómenos como de elecciones estratégicas de los tomadores de decisiones y trazadores de políticas y, por supuesto, también resulta de la manera en que algunos actores del escenario internacional han definido sus intereses o han intervenido incluso en el asunto.

Es particularmente importante hacer el análisis de las repercusiones de este proceso en el escenario vecinal inmediato de Colombia, que podría caracterizarse por los siguientes rasgos:

(1) El sostenido alineamiento de Colombia con los Estados Unidos, en el marco de una región que tiende a elaborar retóricas más autonomistas en política internacional y que no ha sido inmune a cierto antinorteamericanismo en boga en el mundo (Deich, 2008).

(2) La configuración en la región de un arco de solidaridades ideológicas -el "socialismo del siglo XXI"- (Dieterich, 2008), que se ubica en las antípodas políticas del régimen colombiano y que puede tener distintas afinidades, siquiera discursivas, con los grupos narcoterroristas.

(3) La aparición de procesos de reconfiguración de la institucionalidad regional (Unasur). Todo lo anterior, además, mientras los grupos narcoterroristas descubren también la importancia estratégica de las zonas de frontera como retaguardia, y encuentran santuario más allá de ellas (con o sin connivencia de los gobiernos vecinos) ("Capaña 'Juzgar a Chávez' Insta a la Comunidad Internacional a Apoyar a Colombia", 2010). 
La relación con Venezuela es, en el escenario antes descrito, uno de los factores más complejos de perturbación del clima de seguridad y defensa de Colombia en el marco regional. Lo anterior sigue siendo cierto a pesar de los recientes y muy positivos acercamientos entre los dos gobiernos, en una oportuna superación del antagonismo personal que permeó las relaciones bilaterales en la administración Uribe ("Mi nuevo mejor amigo" llamó Juan Manuel Santos a Hugo Chávez", 2010).

En efecto, a pesar del clima de normalización actualmente existente, no se puede incurrir en la ingenuidad de desconocer la naturaleza expansionista del proyecto chavista ni de creer que, de la noche a la mañana (y por muy consciente que esté el presidente Chávez de lo nocivos que resultan sus vínculos con el narcoterrorismo para su propio proyecto de liderazgo continental y regional), el régimen de Miraflores se va a convertir en un aliado activo y comprometido de Colombia en la lucha contra el narcoterrorismo y que abandonará su negligencia cómplice (por no hablar de simpatía activa) hacia las FARC y el ELN (Gaviria, "PSUV y FARC, Organizaciones Hermanas", 2010 ).

A lo anterior cabe añadir el creciente empoderamiento militar de Venezuela y su reciente aspiración a dotarse de un programa de energía nuclear ("Chávez: Venezuela estudia proyecto de energía nuclear con fines pacíficos", 2010). Aunque estos hechos no pueden sobre-dramatizarse, tampoco pueden ser simplemente soslayados en lo que no sería sino un peligroso y tramposo apaciguamiento.

Otro de los frentes críticos a nivel vecinal para la seguridad colombiana es Ecuador. Sin embargo, cabe anotar que en relación con ese país el evento más crítico (el bombardeo al campamento de "Raúl Reyes" en Sucumbíos ("Ejército bombardeó campamento de Raúl Reyes luego de reunión con Piedad Córdoba", 2007) desencadenó un proceso que a largo plazo puede resultar constructivo, a pesar de que se mantengan las diferencias ideológicas entre Quito y Bogotá ("Colombia y Ecuador retoman relaciones diplomáticas", 2010), en tanto que Carondelet siga orbitando alrededor del eje del "socialismo del siglo XXI".

En efecto, el bombardeo de Sucumbíos puso en evidencia, para el propio Gobierno ecuatoriano, el grado de penetración de las FARC en instancias oficiales, obligó a Ecuador a reforzar la seguridad en la frontera (con la excusa de prevenir una nueva incursión colombiana) y, de paso, a hacer frente a las FARC, al menos de vez en cuando, en el terreno militar, sometiendo a revisión el "dejar hacer, dejar pasar" que había practicado durante mucho tiempo ("Militares ecuatorianos destruyeron 126 bases de las FARC durante 2010", 2011).

A ello se añade que la normalización de las relaciones con Quito, con base en la hoja de ruta acordada hace ya algo más de un año entre las dos cancillerías, puede contribuir a la reducción de la hostilidad hacia Colombia en el vecindario y a su nivel de exposición en los foros multilaterales latinoamericanos, en los que el chavismo regional ha querido acorralarla. 
Por otro lado, Colombia tiene el desafío de intervenir activamente en los procesos de reconfiguración institucional regional, con dos prioridades directamente relacionadas con su seguridad:

(1) La preservación del sistema interamericano y su fluida conexión con el emergente sistema Unasur.

(2) La generación de blindajes institucionales frente a potenciales riesgos externos, como por ejemplo, el armamentismo venezolano, la volatilidad del régimen de Chávez, etc.

En efecto, Colombia no puede permitir que por sustracción de materia se desmonte la OEA, ni que la arquitectura de Unasur sea definida en los términos en que Venezuela o Brasil lo desean, al margen de los intereses nacionales colombianos. Al mismo tiempo, debe aprender a apalancarse en esa nueva institucionalidad para conseguir recursos de contención frente a Venezuela, que hasta ahora ha sido fallido obtener en otros escenarios.

\section{Segunda conclusión}

En consecuencia, el país se ha visto en la necesidad de mantener el esfuerzo de normalización con Venezuela, pero sin comprometer el margen de autonomía de Colombia ni mucho menos la seguridad nacional. En ese sentido, el Gobierno tiende, de algún modo, a mantener las precauciones y reservas limitando el avance material de la cooperación para seguridad, hasta tanto existan garantías del compromiso venezolano (sometido a verificación).

Entre tanto, la situación evoluciona mediante acuerdos puntuales y formales destinados a generar y consolidar confianza mientras que, discretamente, refuerzan la normalización en los otros niveles (diplomático y económico, especialmente).

Paralelamente, Colombia ha ido monitoreando y valorando cuidadosamente los alcances e implicaciones del armamentismo venezolano para actuar con tanta prudencia como anticipación en tan comprometedora materia.

En cuanto al Ecuador, Colombia tiende a aceptar, pragmáticamente, que a pesar de su militancia en el 'Socialismo del siglo XXI' (Dieterich, 2008), los propios intereses internos y de gobierno pueden contribuir a la fluidez de la relación bilateral. Además, esa normalización tiende a hacerse necesaria para reducir la vulnerabilidad de Colombia en medio de un vecindario potencialmente hostil, y podría apalancar algunos avances con Chávez. Es por eso que, en materia de seguridad, la cooperación puede llegar a ser incluso más profunda, pero Bogotá tampoco parece esperar de Quito un compromiso radicalmente profundo, pues Ecuador sigue manteniendo la teoría de su "neutralidad" frente al conflicto irregular ("Ecuador mantendrá neutralidad frente a conflicto de Colombia", 2004). 
Todo esto significa que para asegurar que la nueva arquitectura institucional suramericana se defina en términos que respeten el legítimo interés nacional colombiano, y no se convierta en instrumento de las pretensiones geopolíticas de otros Estados (como Venezuela o Brasil), Colombia ha ido asumiendo un rol de liderazgo y proactividad, promoviendo su desarrollo, la creación de instancias técnicas y especializadas en materias de su especial interés y, sobre todo, incidiendo en la definición de la agenda de seguridad suramericana (FES/CEEPADE, 2010).

De hecho, su potencial posicionamiento en el Consejo de Seguridad de la ONU parece contribuir a dotarle de suficiente legitimidad para enlazar la agenda regional y la agenda mundial de seguridad (Rojas, "Santos le apostó a un viraje en la diplomacia", 2011).

\section{TERCERA PARTE: UNA COMPLEJA ARTICULACIÓN A ESCALA GLOBAL}

Uno de los frentes en que más notorio ha sido el impacto de la internacionalización de la problemática de seguridad de Colombia, ocurrida (y también provocada, intencionalmente, tanto por el Gobierno como por las organizaciones armadas ilegales) durante los últimos años es, sin duda, el que tiene que ver con la profundización del alineamiento de Colombia con los Estados Unidos (World of Information, 2006), aunque este alineamiento no sea en modo alguno inédito, pues en la práctica puede remontarse a la participación de Colombia en la Guerra de Corea en 1950 (y no obstante la orientación de la política exterior de gobiernos como el de Alfonso López Michelsen o Belisario Betancur).

Pero lo que es innegable es que nunca antes la alianza entre los dos países había llegado a tener una profundidad y una importancia estratégica (al menos para Colombia) como la que tiene actualmente (Christman \& Heimann, 2004).

Por otro lado, no menos importante resulta la configuración de otros escenarios de internacionalización, que hasta hace algún tiempo parecían totalmente ajenos a la evolución y la dinámica de la seguridad en Colombia. Los nexos profundos de organizaciones como las FARC con la banda terrorista ETA, y de ambas con algunos elementos del régimen chavista de Venezuela ("ETA y FARC, una vieja amistad", 2010), así como la actividad de algunas organizaciones no gubernamentales en Europa en defensa de la causa "fariana" ("La diplomacia de las FARC", 2009), ejemplifican perfectamente este fenómeno de peculiar importancia.

En efecto, la relación entre Colombia y Estados Unidos ha sido caracterizada, con acierto, como una "relación especial", sobre todo si se mira en el contexto general de la región, caracterizado por una tendencia, si no confrontacional, por lo menos de relajamiento del orbitamiento de las naciones latinoamericanas alrededor de Washington y la diversificación creciente de su nómina de interlocutores en la escena internacional (Lowenthal, Piccone, \&Whitehead, 2009). 
Esta relación especial se ha construido alrededor de dos ejes principales. Por un lado, la guerra contra las drogas, planteada por Washington desde el gobierno Nixon y convertida en realidad bajo Reagan, uno de cuyos campos de batalla más importantes ha sido siempre Colombia, en donde los grandes carteles del narcotráfico llegaron incluso a convertirse en la principal amenaza a la seguridad nacional.

Por el otro, a partir de los sucesos del 11 de septiembre de 2001, y en especial bajo las administraciones Bush II y Uribe, la guerra contra el terrorismo. El resultado de la sincrónica y estratégica articulación de estos dos ejes no fue otro que el Plan Colombia, al cual se debe, en buena medida, el éxito obtenido durante la última década por el Estado colombiano en su lucha contra las organizaciones armadas ilegales, rotuladas como "narcoterroristas" (Cragin \& Hoffman, 2003).

Pero no solo el Gobierno colombiano ha buscado en el plano internacional recursos, tanto políticos como económicos, que le permitan apalancar su lucha contra las organizaciones armadas ilegales; estas mismas organizaciones ilegales también, a su manera, han sabido aprovecharse de los beneficios de la globalización, y a partir de la fallida experiencia de los diálogos de San Vicente del Caguán (CLACSO, 2010) -aunque no por causa de ellos- ("Cronología del proceso de paz", 2002), descubrieron, gracias a la cooperación y a la transferencia de aprendizajes (por ejemplo de la red Askapena, brazo internacional de ETA), las ventajas de la diplomacia paralela y la guerra política internacional.

El establecimiento y fortalecimiento de los vínculos de las FARC y el ELN con otras organizaciones de similar naturaleza, como la propia ETA ("Guerrilleros de FARC, ELN y ETA, según diplomáticos de EE.UU. (Wikileaks)", 2010) y también el IRA (ETA adiestra al ELN en técnicas para llevar a cabo actos terroristas, según Ejército colombiano", 2001), le da necesariamente a la lucha antiterrorista que libra el Estado colombiano una proyección internacional que implica la configuración de nuevos campos de acción que no pueden soslayarse, y para intervenir en los cuales es preciso recabar y obtener de manera eficiente y eficaz el apoyo y la cooperación de otros gobiernos.

Esta expansión internacional de las organizaciones armadas ilegales ha sido posibilitada también por su capacidad para difundir propaganda e instrumentalizar en provecho de su causa a algunas organizaciones no gubernamentales, que muchas veces actúan como idiotas útiles al servicio de la promoción de los intereses del narcoterrorismo ("La 'diplomacia' de las FARC", 2009). En otros casos, sin embargo, las organizaciones armadas ilegales han desarrollado sus propias organizaciones no gubernamentales, e incluso empresas de fachada, por medio de las cuales libran la guerra política y jurídica contra el Estado colombiano.

Estas organizaciones operan de manera transnacional, tienen una enorme influencia en los medios de comunicación, están de alguna manera blindadas por su pretendida vocación filantrópica y tienen un increíblemente amplio margen de interlocución y de acción ante otros gobiernos y 
ante la opinión pública internacional y, por lo tanto, constituyen hoy por hoy uno de los centros de gravedad de la amenaza en Colombia.

\section{Tercera conclusión}

En consecuencia, el Gobierno ha constatado la importancia de mantener la relación particular y especial con los Estados Unidos. Dicha relación apalanca no solo la capacidad disuasoria del Estado colombiano frente a las organizaciones narcoterroristas internas, sino también frente a potenciales agresiones externas y, en particular, frente a la potencialmente hostil actividad del régimen chavista de Venezuela (World Information, 2006).

Sin embargo, también parece comprender que esa relación está sujeta a coyunturas y a factores de cambio muy complejos y no puede darse por sentada, en la medida en que, por ejemplo, se vaya agotando el ciclo vital del Plan Colombia, se desplace el frente de batalla antidrogas (a México y Centroamérica) o varíe la retórica (no la práctica) de Washington en relación con la lucha contra el terrorismo.

Por otra parte, y a pesar de la normativa internacional existente, la mayor parte de las veces la cooperación internacional en la lucha contra el terrorismo parece insuficiente. Sin embargo, el Gobierno ha visto que es posible aprovechar las coincidencias específicas que puedan existir en las agendas de seguridad de otros Estados (por ejemplo con España en el caso de ETA), para diseñar una estrategia tanto de sincronización inmediata y directa de esfuerzos como de presión subsidiaria y transitiva, esto es, proporcionar a España, por ejemplo, suficiente material probatorio y cooperación para que sea Madrid, y no Bogotá, quien presione a Caracas a efectos de neutralizar el perverso maridaje del régimen chavista con las organizaciones narcoterroristas FARC y ETA.

Por último, el Estado colombiano se muestra notablemente interesado en desplegar una estrategia doble en relación con la actividad de propaganda y cooperación que algunas ONG realizan en distintos escenarios internacionales a favor de las FARC y del ELN ("No podemos permitir una diplomacia paralela: Presidente Uribe", 2010).

Para ello, parece ser cada vez más consciente de la necesidad de pasar de la retórica de la condena indiscriminada (usada bajo la administración Uribe), relativamente contraproducente e ineficaz, a una acción comunicacional para contrarrestar la propaganda subversiva. Por tanto, ello obliga progresivamente al Estado a desarrollar campos poco explorados hasta ahora: la diplomacia pública, la denuncia internacional selectiva y discriminada de las actividades del narcoterrorismo y la difusión sincronizada ante la opinión pública de otros países de la magnitud de la amenaza y su ensañamiento con la población civil, en contraste con el esfuerzo legítimo, transparente y legal del Estado colombiano para defender y garantizar la seguridad democrática y el respeto de los derechos y libertades de todos los ciudadanos. 


\section{BIBLIOGRAFÍA}

- $\quad$ (2010, 23 de julio). Campaña 'Juzgar a Chávez' Insta a la Comunidad Internacional a Apoyar a Colombia. EL Tiempo, Disponible en: http://www.eltiempo.com/archivo/documento/CMS-7822109

- (2010, 18 de julio). Chávez amaga con romper relaciones con Colombia. El País. Disponible en: http:// www.elpais.com/articulo/internacional/Chavez/amaga/romper/relaciones/Colombia/elpepiint/ 20100718elpepiint_9/Tes,

- $\quad$ (2010, 28 de septiembre). Chávez: Venezuela estudia proyecto de energía nuclear con fines pacíficos. Noticias Diarias. Disponible en: http://noticiasdiarias1.wordpress.com/2010/09/28/chavez-venezuelaestudia- proyecto-de-energia- nuclear-con-fines-pacificos/ .

- Christman, D. W \& Heimann, J. G. (2004). Andes 2020: A New Strategy for the Challenges of Colombia and the Region: Report of an Independent Commission Sponsored by the Council on Foreign Relations Center for Preventive Action. Council on Foreign Relations . Nueva York, NY, EE.UU. 18-21, Disponible en: http://site.ebrary.com/lib/urosario/Doc?id=10050752\&ppg $=20$.

- Clacso. (2010). OSAL - Observatorio Social de América Latina, Número 7, 94-100. Disponible en: http://site.ebrary.com/lib/urosariosp/Doc?id=10384258\&ppg=99.

- $\quad$ (2010, 27 de noviembre). Colombia y Ecuador retoman relaciones diplomáticas. El País. Disponible en: http://www.elpais.com/articulo/internacional/Colombia/Ecuador/retoman/relaciones/diplomaticas/ elpepuint/20101127elpepuint 1/Tes .

- Cragin, K. \& Hoffman, B. (2003). Arms Trafficking and Columbia. Santa Mónica E.E.U.U.: RAND Corporation.

- (2002, 21 de febrero) Cronología del proceso de paz. BBC Mundo. Disponible en: http://news.bbc.co.uk/ hi/spanish/latin_america/newsid_1752000/1752115.stm.

- Deich, F. (2008). Bolivarismo. Observatorio de Venezuela. Número 2, Año I. Disponible en: http:// site.ebrary.com/lib/urosariosp/Doc?id=10405897\&ppg=19

- $\quad$ Dieterich, H. (2008). Hugo Chávez y el socialismo del siglo XXI. Segunda edición. Argentina: El Cid Editor. Disponible en: http://site.ebrary.com/lib/urosariosp/Doc?id=10219515\&ppg=33

- $\quad$ (2004, 17 de marzo) Ecuador mantendrá neutralidad frente a conflicto de Colombia. Caracol. Disponible en: http://www.caracol.com.co/nota.aspx?id=119410.

- $\quad$ (2007, 9 de noviembre) Ejército bombardeó campamento de Raúl Reyes luego de reunión con Piedad Córdoba. Caracol. Disponible en: http://www.caracol.com.co/nota.aspx?id=504388.

- $\quad$ (2001, 19 de agosto) ETA adiestra al ELN en técnicas para llevar a cabo actos terroristas, según Ejército colombiano. El País. Disponible en: http://www.elpais.com/articulo/internacional/ETA/adiestra/ELN/ tecnicas/llevar/cabo/actos/terroristas/Ejercito/colombiano/elpepuint/20010819elpepuint_3/Tes 
- (2010, 2 de marzo) ETA y FARC, una vieja amistad. Semana. Disponible en: http://www.semana.com/ noticias-conflicto-armado/eta-farc-vieja-amistad/135744.aspx .

- $\quad$ Fundación Friedrich Ebert Stiftung (FES) \& Consejo de Defensa Suramericano y Conducción Política de Defensa. Centro de Estudios Estratégicos para la Defensa (Ceepade), (2010). Publicación del Ministerio de Defensa de la Nación. República Argentina. Extraído febrero 5, 2011, desde http://issuu.com/ceepade/ docs/jornadas fes_ceepade-2010-low

- $\quad$ Fundación Seguridad y Democracia. (2007). La seguridad en los últimos tres periodos presidenciales 1994-2006. Fundación Seguridad y Democracia. Informe especial. Bogotá Colombia.

- $\quad$ Fukumi, S. (2008). Cocaine Trafficking in Latin America: EU and US Policy Responses. Abingdon, Oxon, GBR: Ashgate Publishing Group. Disponible en: http://site.ebrary.com/lib/urosario/Doc?id= $\underline{10215572 \& p p g=186}$

- García, M. (1992). De La Uribe a Tlaxcala : Procesos de Paz. Editorial Cinep. (20-54 p.). Bogotá, Colombia.

- $\quad$ Gaviria, J: (2010, 28 de julio). PSUV y FARC, Organizaciones Hermanas. El Tiempo. Disponible en: http://www.eltiempo.com/archivo/documento/MAM-4071867

- $\quad$ (2010, 30 de noviembre) Guerrilleros de FARC, ELN, y ETA según diplomáticos de EE.UU. (Wikileaks) EI Tiempo. Disponible en: http://www.eltiempo.com/mundo/estados-unidos/ARTICULO-WEBNEW_NOTA INTERIOR- $\quad \underline{8490565 . h t m l}$

- Kline, H. F. (2007) Chronicle of a Failure Foretold: The Peace Process of Colombian President Andrés Pastrana. Tuscaloosa, E.E.U.U: University of Alabama. Disponible en: http://site.ebrary.com/lib/urosario/ Doc?id=10218366\&ppg $=62$

- $\quad$ (2009, 11 de junio) La 'diplomacia' de las FARC. Cambio. Dsiponible en: http://www.cambio.com.co/ portadacambio/831/ARTICULO-WEB-NOTA_INTERIOR_CAMBIO-5346592.html .

- Lowenthal, A. F., Piccone, T.\& Whitehead, L. (Eds.). (2009). Obama Administration and the Americas: Agenda for Change. Washington, DC, EE.UU: Brookings Institution Press. Disponible en: http:// site.ebrary.com/lib/urosario/Doc?id=10338443\&ppg=226

- (2011, 16 de enero). Militares ecuatorianos destruyeron 126 bases de las FARC durante 2010. El Espectador. Disponible en: http://www.elespectador.com/noticias/elmundo/articulo-245196-militares-ecuatorianos-destruyeron-126-bases-de-farc- durante-2010.

- $\quad$ (2010, 7 de noviembre). 'Mi nuevo mejor amigo' llamó Juan Manuel Santos a Hugo Chávez. El Tiempo. Disponible en: http://www.eltiempo.com/archivo/documento/CMS-8302260 .

- $\quad$ (2010, 30 de julio) No podemos permitir una diplomacia paralela: Presidente Uribe. El Colombiano. Disponible en: http://www.elcolombiano.com/BancoConocimiento/N/no_podemos_permitir una_diplomacia_paralela_presidente_uribe/no_podemos_permitir_una_diplomacia_paralela_ presidente uribe.asp 
- $\quad$ Pärssinen, Martti \& Lammila (Eds.). (2006). Colombia Proceso de paz en el 2006. Helsinki, Finlandia Instituto Renvall, Universidad de Helsinki.

- $\quad$ Presidencia de la República, Ministerio de Defensa Nacional. (2003). Política de Defensa y Seguridad Democrática. Extraído Marzo 15, 2011, desde http://www.presidencia.gov.co/seguridad_democratica.pdf.

- $\quad$ Rangel, A. (2007). Sostenibilidad de la seguridad democrática. Colombia: Fundación Seguridad y Democracia. Disponible en: http://site.ebrary.com/lib/urosariosp/Doc?id=10165704\&ppg=20

- $\quad$ Rojas, D. M. (2009). Plan Colombia II: ¿más de lo mismo? Colombia: Red Colombia internacional. Disponible en: http://site.ebrary.com/lib/urosariosp/Doc?id=10337861\&ppg=5.

- $\quad$ Rojas, D. (2011, Impreso No. 139). Santos le apostó a un viraje en la diplomacia. UN Periódico. Disponible en: http://www.unperiodico.unal.edu.co/dper/article/santos-le-aposto-a-un-viraje-en-la-diplomacia/

- Van Engeland, A.\& Rudolph, R. M. (2008). From Terrorism to Politics. Abingdon, Oxon, GBR: Ashgate Publishing Group. Disponible en: http://site.ebrary.com/lib/urosario/Doc?id=10228260\&ppg=150 5. Feb.11.

- World Information. (2006). Colombian Profile. Cambridge, Inglaterra: Walden Publishing Ltd. Disponible en: http://site.ebrary.com/lib/urosario/Doc?id=10120800\&ppg=2 1. Feb. 11. 